\title{
Mucopolysaccharidoses and Orthopedic Management (Focused also on Craniocervical Junction)
}

\author{
Christina Lampe ${ }^{1} \quad$ Christian Gerhard Lampe ${ }^{2}$ \\ ${ }^{1}$ Center for Rare Diseases, Clinic for Pediatric and Adolescent \\ Medicine, Helios Dr. Horst Schmidt Clinics, Wiesbaden, Germany \\ ${ }^{2}$ Clinic for Pediatric and Adolescent Medicine, Helios Dr. Horst \\ Schmidt Clinics, Wiesbaden, Germany \\ J Child Sci 2018;8:e128-e137.
}

\begin{abstract}
Address for correspondence Christina Lampe, MD, Center for Rare Diseases, Clinic for Pediatric and Adolescent Medicine, Helios Dr. Horst Schmidt Clinics, Ludwig-Erhard-Street 100, 65199 Wiesbaden, Germany (e-mail: christina.lampe72@gmail.com).
\end{abstract}

\begin{abstract}
Keywords

- musculoskeletal

- joints

- craniocervical junction

- mucopolysaccharidoses

- dysostosis multiplex

Mucopolysaccharidoses (MPSs) are multisystemic, chronic progressive, heterogeneous, and life-threatening diseases, involving severely the musculoskeletal system, in particular in MPS I, II, IV, VI, VII, and less prevalent in MPS III. Accumulation of glycosaminoglycans (GAGs) in soft tissues, such as ligaments, tendons, and joint capsules, as well as in cartilage, and bone lead to orthopedic complications: joint stiffness, contractures, and skeletal deformities, resulting in hip dysplasia, genua valga, feet deformities, kyphoscoliosis, narrowing of the spinal canal, atlantoaxial instability, carpal tunnel syndrome, trigger finger, and growth retardation. These complications significantly reduce the quality of life due to impaired mobility, loss of independency, and pain. Compression of the cervical spinal cord is also life threatening. According to the progressive nature of MPSs, musculoskeletal symptoms worsen over time and surgical intervention may be inevitable. However, due to the increased anesthesia risk of MPSs, surgical intervention has to be evaluated carefully by a multidisciplinary team. Additionally, due to the rarity of the diseases, not many standards or recommendations are available to determine the indication of a surgical intervention, so each intervention must be decided individually, based on the few data available. In addition, conservative treatment should be taken into consideration. Physiotherapy, pain medication, insoles, orthosis, braces, corsets, and special footwear play a pivotal role. Unfortunately, the skeletal tissues are poorly vascularized and enzyme replacement therapy does not have much effect on them. It is important to detect and observe musculoskeletal complications in the regular follow-up visits, in particular the life-threatening compression of the cervical spinal cord.
\end{abstract}

\section{Introduction}

Skeletal and joint involvements are the major disease manifestations in MPSs. This is evidenced by looking at the classification of skeletal dysplasias (SD) according to the

received

July 3, 2018

accepted after revision

July 17,2018
Issue Theme Advances in

Mucopolysaccharidoses; Guest Editors: Susanne G. Kircher, MD, PhD, MBA, Adriana M. Montaño, $\mathrm{PhD}$, and Christina Lampe, MD

DOI https://doi.org/ 10.1055/s-0038-1669384. ISSN 2474-5871. clinical, radiological, and genetic information. One of those categories corresponds to the MPSs which are classified as "lysosomal storage diseases with skeletal involvement (dysostosis multiplex group)."1 The importance of skeletal involvement in MPSs is also shown historically. In the 1960s,
Copyright $\odot 2018$ Georg Thieme Verlag License terms KG Stuttgart - New York 
before elucidating the genetic background and pathogenesis of various diseases, skeletal dysplasias were divided in two groups: achondroplasia, "short-limbed type of SD" and Morquio syndrome, "short-trunked type of SD." Now, it is known that not only skeletal dysmorphism, but also glycosaminoglycan (GAG) accumulation in tendons, ligaments, joint capsules, and cartilage are leading to structural deformities and loss of function of the joints.

\section{Mechanism of Bone and Joint Disease}

Joint disease in the MPSs is progressive and typically without clinical signs of inflammation. While MPS III have less joint involvement, MPS I, II, VI, and VII patients suffer from joint stiffness and contractures. In contrast, MPS IV patients show hypermobility of joints and usually more severe skeletal dysplasia than the other MPS types. As a result, also osteopenia and joint destruction has been described. ${ }^{2}$

The GAG storage in articular cartilage leads to enhanced chondrocyte apoptosis, increased nitric oxide production, increased cytokine and chemokine production, macrophage recruitment, activation of TLR4 pathway, and increased matrix metalloproteinases (MMPs) and TNF- $\alpha{ }^{2}$ Moreover, GAG storage in growth plate leads to altered growth plate morphology, altered trabecular architecture, osteoclast dysfunction, inhibition of collagenase activity of cathepsin $K$, alteration of the STAT pathway, and decreased IL-6 and IL-6 family cytokines.

In summary, although not having any clinical signs of joint inflammation on a cellular basis, there are many different inflammatory pathways ongoing due to GAG deposition, but not all mechanisms are understood so far. ${ }^{2}$

\section{Dysostosis Multiplex}

The radiological term "dysostosis multiplex" is characteristic for MPS and reflects defective endochondral and membranous bone formation and maturation throughout the body, including the following skeletal dysmorphisms:

- Skull: J-shaped sella turcica, thickened calvaria.

- Chest: Oar shaped ribs, shortened sternum, broad-short clavicles.

- Spine: Platyspondyly, inferiorly beaked vertebrae, odontoid dysplasia.

- Pelvis: Round iliac wings, inferior ilial tapering, hip dysplasia.

- Hands: Proximal pointing of metacarpals, shortened metacarpals, hypoplasia of carpals.

- Long bones: Hypoplastic epiphyses, thick-short diaphysis. ${ }^{2}$

Heterogeneity of disease progression and disease severity in each MPS type reflects the degree of dysostosis multiplex. Thus, there are some skeletal/joint disease complications that are more frequent than others and need sometimes surgical intervention. In any case intensive physiotherapy, stretching of the joints/ligaments and tendons, orthosis, insoles, and corsets, as well as individualized footwear may improve and maintain mobility, and reduce pain.

Spinal cord compression on the level of the craniocervical junction is a life-threatening musculoskeletal disease complication. In contrast, hip dysplasia, feet deformities, and kyphoscoliosis reduce mobility, evoke pain, and reduce quality of life (QOL).

Regular evaluation of skeletal and joint disease and followup visits are important. Regular clinical examinations, measurement of joint range of motion, X-rays, CT (computed tomography) scans, and MRIs (magnetic resonance imagings, especially of the spine) may help to understand the disease progression and need of surgical intervention. In particular, the decision for surgical intervention must be analyzed very carefully due to the high anesthesia risk in MPSs.

\section{Lower Limb Involvement}

Lower limb involvement such as hip dysplasia, genua valga, ankle and foot deformities, carpal are very common orthopedic disease complications in MPSs. Orthopedic findings in different MPS types ${ }^{3}$ and recommendations of radiological assessments in MPSs have been published. ${ }^{4}$ Currently, recommendations for follow-up assessments are not suggested for all MPS types.

Further investigations to analyze the lower limbs in MPS are described by White et al, regarding Morquio patients but might be adapted to other MPSs (- Fig. 1). ${ }^{5}$

- Physical examination: At diagnosis, annually and when clinically indicated.

- Anteroposterior pelvis X-ray: At diagnosis, annually and when clinically indicated.

- Anteroposterior standing radiographs of both legs: At diagnosis, when clinically indicated.

- CT for rotational alignment or preoperative: when clinically indicated.

- MRI: When clinically indicated.

- Arthrography: When clinically indicated.

- Gait analysis: When clinically indicated.

\section{Hip Dysplasia}

Hip dysplasia is found in almost all children with MPS. Typical changes of the hips include the loss of sphericity of the femoral head, flat acetabula, increased neck-diaphyseal angle, and migration of the hip. The result is impaired mobility and pain, although many radiological changes do not correlate with the clinical symptoms.

An international consensus procedure concerning the treatment of the hip dysplasia in MPS I patients, after Hematopoietic Stem Cell Transplantation (HSCT), recommends that an early corrective surgery should be considered, but further research is needed to establish its efficacy. There was no consensus whether surgical correction should be offered to all or only to symptomatic patients. ${ }^{6}$

In 2016, Kennedy et al published "Along-term retrospective evaluation of functional and radiographic outcomes of pediatric hip surgery in Hurler Syndrome”. In this study, 13 MPS I patients underwent 24 hip surgeries. The average age at surgery was 4 years (2-6.3 years). The average follow-up was 14.6 years (10.3-21.6 years). 12 out of 13 patients underwent a Salter pelvic osteotomy \pm proximal femoral osteotomy. In the follow-up, $41 \%$ showed advanced degenerative changes, although radiological well covered hips, 


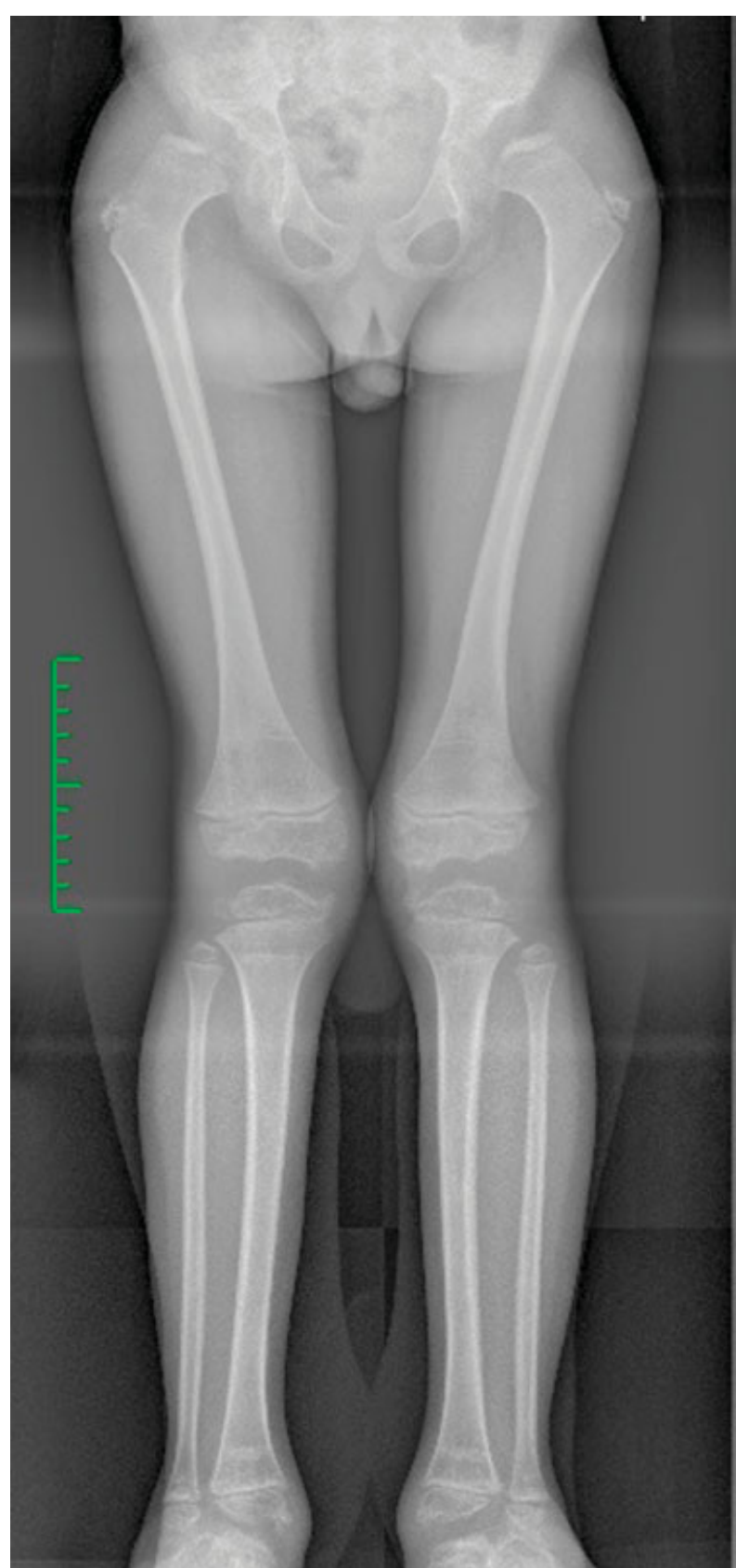

Fig. 1 X-ray of both legs in an 8-year-old male Morquio A patient with hip dysplasia, genua valga, and deformity of the ankles.

evaluated by the "Harris hip score". ${ }^{7}$ Due to this scoring system, 7 out of 13 cases were analyzed as surgical failures. It seems that radiological findings and clinical outcome do not correlate. $^{8}$

A retrospective study, analyzed 88 hips in 44 children with MPS I and II to understand the hip morphology. X-rays evaluated hip migration, femoral head sphericity, and acetabular dysplasia at different ages throughout childhood. 75\% of children showed progressive hip migration, and 50\% progressive femoral head deformity, while the acetabulum varied from normal to severely dysplastic but did not deteriorate over time. MPS I patients seemed to be more affected than those suffering from MPS II. According to the fact, that some of the above-mentioned changes of the hips did not progress over time; the authors suggest that surgery was not needed in all cases. ${ }^{9}$

The evaluation of 124 out of 505 MPS II patients, enrolled in the Hunter Outcome Survey, showed in 30 patients with available X-rays of the hips the presence of any hip abnormality in 26 patients, mainly acetabulum dysplasia followed by femoral head dysplasia. Pelvic osteotomy or hip replacement was not reported in any patient, only 3 patients received femoral osteotomy. ${ }^{10}$

Although MPS III patients do not show severe signs of dysostosis multiplex as other MPS types, they may show osteonecrosis of the femoral head: a Dutch study analyzed the X-rays of 33 MPS III patients. Fourteen patients were considered as attenuated affected. In these patients, no osteonecrosis of the femoral head was detected. In contrast to that, osteonecrosis of the femoral head was described unilateral in 8 severely affected patients and bilateral in 6 patients. Additionally, 6 patients had also hip dysplasia. ${ }^{11}$

The physicians survey of 56 MPS VII patients reported that $53 \%$ suffered from hip dysplasia, and around $25 \%$ had hip replacement. Hip dysplasia was the main reason for losing ability to walk in this cohort. ${ }^{12}$

In a prospective study, over 2 years in 14 MPS VI patients, $157 \mathrm{X}$-rays were evaluated. Median follow-up was 6.8 years. In all patients, dysplasia of the os ilium and the acetabulum was described. The femoral head appeared normal in young children, but worsened over time due to impaired ossification. Although the acetabular coverage of the femoral head improved over time, it still remained insufficient. It was concluded that all patients suffered from structural hip deformities over time that vary from patient to patient but led to different degrees of impaired mobility in all. However, this progression is difficult to predict. ${ }^{13}$

Another study was enrolled 23 MPS IVA patients. Hip subluxation was seen in all patients, and 61 hip surgeries were performed in these patients. Six patients (12 hips) had recurrence of subluxation after acetabular osteotomies and/ or femoral varus derotation osteotomy. ${ }^{14}$

Since the acetabulum is not capable to remodel, the acetabular cartilage in MPS patients differs from the "common dysplasia of the hip." Thus, femoral osteotomy in combination with pelvic osteotomy may lead to dislocation while femoral osteotomy combined with acetabuloplasty may avoid dislocation. ${ }^{14,15}$

However, these are invasive procedures with long rehabilitation time and long immobility. Additionally, the high anesthesia risk in MPS patients must be taken into consideration and balance carefully risk and benefit. Another surgical option is total hip replacement, but this procedure should be reserved for adult patients.

Beside the skeletal deformities of the hip, also contractures of the hip flexors lead to inappropriate biomechanical stress to the femoral head, the spine, and all other joints of the limbs. To stand upright and walk stable, the body tries to balance the flexion in the hips with hyperlordosis of the lumbar spine, flexion of the knees, and finally tip toe walking. On the other hand, the contractures of the knees 
in MPS II appear with median onset at 5.1 years while the median age of onset of hip contracture was 5.6, years. ${ }^{10}$ This shows that the musculoskeletal system is very complex and has to be seen as a whole, especially when deciding on surgical interventions. In any case, intense physiotherapy including stretching of the hip adductors may improve the symptoms.

\section{Genua Valga}

Genua valga are very common skeletal symptoms in MPSs, found in almost all children with MPS IV and 50\% of children with MPS I after HSCT. ${ }^{16}$ Valgus deformity is also described in MPS VI, and slowly progressing MPS I (Hurler-Scheie, Scheie), as well as in MPS II.

The best way to evaluate lower extremity alignment is the AP X-ray of both limbs on an image in standing position. It should be assessed at least at first assessment and in the follow-up according to the clinical examination. The following conditions should be analyzed ${ }^{4}$ :

1. Lateral femoral angle (LDFA): Normal averages $88^{\circ}$ (range: $\left.85-90^{\circ}\right)$.

2. Medial proximal tibial angle (MPTA): Normal averages $87^{\circ}$ (range:8 5-90 ${ }^{\circ}$ ).

3. Mechanical axis deviation as graded by zone. ${ }^{4}$

In MPS, the deformity is mainly a valgus of the tibia of more than $90^{\circ}$ and a zone 2 mechanical axis deviation. ${ }^{4}$

Surgical techniques to fix valgus deformities are hemiepiphyseal growth modulation by implantation of Blount's staples or eight plates, as well as classical osteotomy, and in a final stage, knee arthroplasty. Another option is a correction by external frame, especially when mainly the tibia is affected. The less invasive method is the eight-plate implantation that can be used also in very young children. However, the eight-plate technique requires a certain growth to balance the deformity, but growth in MPS cannot be predicted. $^{4}$

The indication for surgical intervention is described as a tibial-femoral angle of more than $15^{\circ} .4$

Fifty-eight knees of 17 MPS I and 12 severe MPS II patients were observed in a retrospective observational study, regarding knee alignment. All patients with MPS I and 75\% of children with MPS II showed genua valga deformity at the age of 8 years with deterioration over time in 66\% in MPS I and $50 \%$ in MPS II patients. Seven children with MPS I, and 3 children with MPS II were treated with implantation of eight plates. The deformity persisted in one patient, recurred in three children after removing the eight plates, and plates were still in place in four children. The plates remained in situ for approximately 1.6 years.

In conclusion, although recurrence is quite frequent, the alignment of genua valga deformity should be considered in progression of valgus deformity in MPS I and II patients. Skeletal maturity prior removal of the eight plates may avoid recurrent deformity having reached skeletal maturity before removing the devices. These results are comparable with another study in eight MPS I knees, showing complete correction in two knees, incomplete correction in six knees, and a recurrent deformity in five knees, after removal of the staples.

In contrast to the stiff joints in MPS I, II, VI, and VII patients, MPS IVA patients show a hypermobility of joints due to laxity of ligaments. Also, the collateral ligaments of the knees are affected. In MRI, the proximal lateral portion of the tibia appears to be unossified and the fibula to be short.

In a study of 23 MPS IVA patients who received different hemiepiphysiodesis techniques at a mean age of 8.3 years, 19 patients remained mobile, $30 \%$ showed improvement in the 6-minute walk test (6MWT) and 3 patients showed immobility without obvious medical reason in an average followup of 44 months. $^{8}$

The only case report of an MPS VI patient indicates that genu valgum is a complication in this disease. ${ }^{4,17}$

There is no literature about MPS III and genua valga.

In a physicians' survey of 56 MPS VII patients, it was reported that 27 patients (63\%) suffered from genua valga. ${ }^{12}$

\section{Feet Deformities}

Feet deformities in MPSs are not well described in literature. Typical deformities in MPSs are pes equinus, hindfoot valgus, forefoot adductus with prominence of the first metatarsal head and curly toes. Additionally, ankle valgus is quite common. The treatment is mainly conservative with insoles, orthotics, and custom footwear. Only one study evaluates the QOL and function after foot and ankle surgery in 18 MPS I patients who have received HSCT (mean age: 10.3 years). Validated questionnaires, with a score of 60 defined as healthy, were used to understand physical, school, play, and emotional domains, as well as footwear habits. The average score for MPS I patients was 45.7. Functional domains had lower scores than other domains like school, play, or emotional aspects. Ten patients were unable to wear common shoes. ${ }^{18}$

White et al described that foot and ankle deformities in MPS IVA patients are usually managed by orthosis, but also by surgical correction with guided growth (screw hemiepiphysiodesis or osteotomy) might be considered, although deformities recur often. ${ }^{5}$

\section{Spine Deformities}

Spinal cord compression is due to spinal deformities and GAG accumulation in ligaments and soft tissues occurs in all levels of the spinal cord, although mainly in the region of the craniocervical junction and the thoracolumbar spine. (-Fig. 2) Since compression of the cervical spinal cord reduces the QOL, which has a big impact on mortality in MPS patients, it is important to screen for and to treat before irreversible organ damage occurs. Taking into consideration, the high anesthesia risk in MPSs, the right time for surgery is of great importance.

The following assessments are needed to detect spinal complications in MPSs:

\section{Neurological Examination}

The first sign of cervical spinal cord compression is loss of endurance and instability in gait. Additionally, patients show pyramidal tract signs like increased or side different deep tendon 


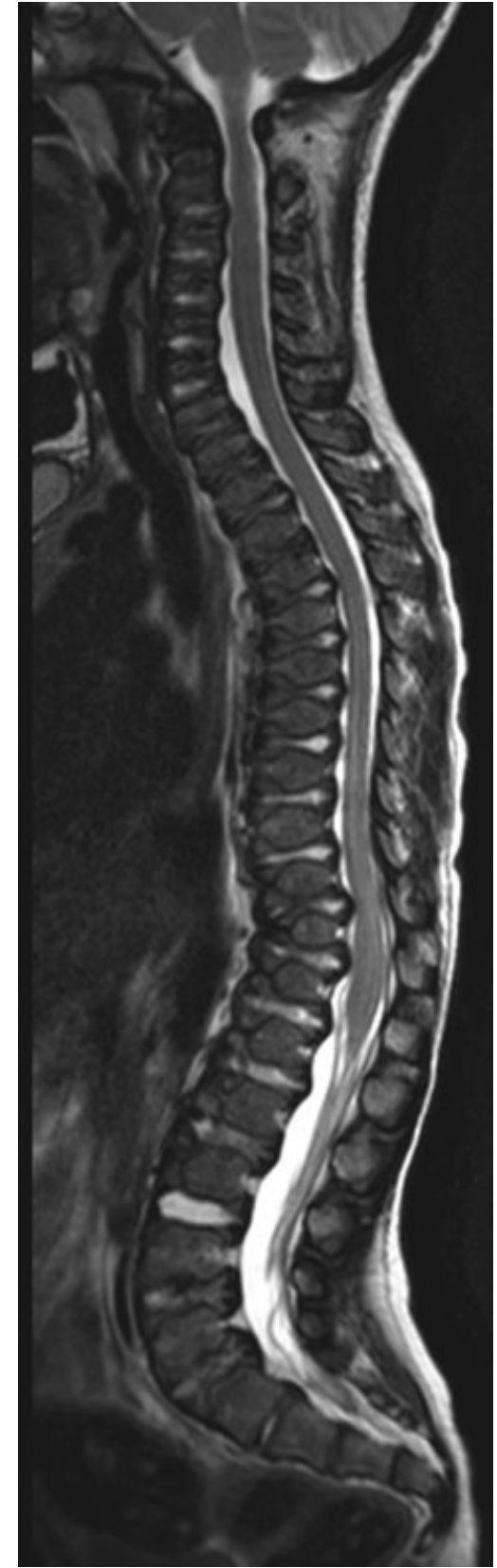

Fig. 2 Magnetic resonance imaging of the whole spine in an 8-yearold male Morquio A patient with narrowing of the spinal cord at the craniocervical junction and vertebral deformities.

reflexes, ankle clonus, and Babinski's sign. Further signs can be loss of strength and raised muscle tone. The lower limbs (legs) are more affected than the upper limbs (arms). Sensory loss, loss of vibration sense, and deep sensation are less common. ${ }^{19}$

Due to the fact that compression of the spinal cord at the thoracolumbar level causes similar neurological signs, a coexistence of both thoracolumbar and cervical spinal cord compression must be excluded.

\section{Imaging}

Several imaging assessments are available to analyze spinal cord compression: MRI, X-ray, and CT scans. ${ }^{19,20}$
In the multinational, multidisciplinary consensus for the diagnosis and management of spinal cord compression among patients with MPS VI, ${ }^{20}$ it is recommended to ask the following questions to the radiologist:

1. Presence, absence, or dysplasia of the odontoid process of $\mathrm{C} 2$, posterior elements, vertebral bodies, and discs.

2. Degree of ligamentous and dural thickening.

3. Spinal column kyphosis, scoliosis, instability, stenosis, or listhesis.

4. Complete or partial effacement of spinal fluid around the cord.

5. Spinal cord contour changes and degree of compression.

These questions are independent from the MPS type and should be used for all MPSs

\section{Magnetic Resonance Imaging}

To detect spinal stenosis, myelopathy or myelomalacia, T1 - and T2-weighted MRI of the whole spine is of upmost importance.

Magnetic resonance imaging is indispensable for soft tissue evaluation (myelopathy and myelomalacia) and it shows venous collaterals. Flexion-extension MRI immediately shows cord compression due to instability. The advantage is also the nonionizing radiation, although sedation is needed in young children and neurological MPS patients that contain the risk of anesthesia complications.

Further techniques, including cisternography, CSF (cerebrospinal fluid) flow, DWI (diffusion weighted imaging), and DTI (diffusion tensor imaging) can be performed for better evaluation and staging of stenosis and potential cord compression. ${ }^{20}$

\section{X-ray}

Further information is given by $\mathrm{X}$-rays, plain and in flexion and extension to detect skeletal deformities, instability, spinal canal stenosis, as well as malalignment. X-ray, in AP and lateral of the whole spine gives an impression of the bony structure of the spine, malformations and additional deformities, such as kyphosis. X-ray, in flexion and extension gives the option to detect instability, although it is sometimes difficult to analyze due to the fact that basilar invagination and enlarged mastoid processes often overlap the craniocervical junction.

\section{Computed Tomography Scan}

Computed tomography scan of the areas of interest not only provides further information about the bony structure, but also it is needed for planning surgical intervention. In particular, it assists in the evaluation of the odontoid process as well as the atlantoaxial junction. It may replace MRI in patients with high anesthesia risk since also flexion and extension studies can be performed and CT scan allow visualization of some soft tissue structures. One disadvantage is the recurrent use of ionizing radiation.

\section{Neurophysiology}

Somatosensory evoked potentials (SSEPs) of the median nerve have been described to be useful in diagnosing and monitoring cervical cord compression in MPS VI. ${ }^{21,22}$ In contrast to that, a 
study of 22 MPS IVA patients found no correlation between neurological examination and SSEP of the median nerve. ${ }^{23}$ By performing SSEPs of the median and the posterior tibial nerves, it could be possible to discriminate between lesions at the level of the craniocervical junction and below the lower cervical spinal cord (Lampe, unpublished data). Motor evoked potentials (MEP) have not been systematically studied in MPSs so far. Intraoperative neurophysiological observation is strongly recommended. ${ }^{19}$

\section{Compression of the Spinal Cord at the Craniocervical Junction}

Beside all mentioned QOL reducing skeletal problems, the craniocervical stenosis is a life-threatening complication. It is very common in MPS IV, VI, less frequent in MPS II and VII, and rare in MPS III.

For MPS IVA and MPS VI patients, recommendations are available ${ }^{19,20}$ on how to detect, follow-up, and manage/find the right time for decompression surgery of the craniocervical stenosis.

The cause of craniocervical stenosis is multifactorial and leads to impairment of movement, paraplegia-mainly of the lower limbs, respiratory insufficiency, central apnea, and tetraplegia leading finally to death.

Anatomically, atlantoaxial instability is due to odontoid dysplasia in combination with ligamentous laxity, in particular of the transverse ligament of the atlas. As a consequence, there is ligamentous hypertrophy and invagination of the posterior arch of $\mathrm{C} 1$ leading to narrowing of the spinal cord, in particular during flexion. Additionally, due to GAG accumulation, thickening of the dural and paraspinal ligaments deteriorate the spinal compression. ${ }^{24}$

The main purpose of the evaluation is to detect presence of spinal cord compression before irreversible spinal cord damage is present. The following assessments may help to diagnose myelopathy: neurological examination, imaging (MRI, CT, X-rays), and neurophysiological examinations. Only the combination of the mentioned examinations allows to analyze the severity of the spinal cord compression and to decide whether surgical intervention is needed. ${ }^{19,20}$

Further examinations, such as sleep studies, pulmonary function tests, ultrasound evaluation of diaphragmatic motion to assess phrenic nerve function, urodynamics, and $6 \mathrm{MWT}$ also help to analyze the function of the spinal cord. ${ }^{20}$

Indication for surgery is given by presence of pathological reflexes, and/or neurological deficits, and signal changes of the spinal cord seen in the MRI. Deterioration of endurance in the 6MWT, central apneas in sleep studies, paresis of the diaphragm or pain in the neck may underlay the need of surgery. Prophylactic decompression was recommended previously but due to the fact that GAG accumulation is progressive, recurrent surgeries may be needed, and patients with MPS have a high anesthesia risk. The aim of decompression surgery is to protect the spinal cord and correct spine malalignment as well as stabilizing instability. ${ }^{19}$

The only therapeutic option is surgical decompression by laminectomy with or without stabilization. There is no consensus about the surgical technique in detail so far. Most common is the posterior fixation and fusion of C1-C2 with instrumentation and bone graft. Several approaches are used and none can be called the gold standard so far. Spinal stenosis can be assumed, if the anteriorposterior diameter of the spinal canal is $<14 \mathrm{~mm}{ }^{19}$

\section{Central Cord Syndrome in MPS IV}

Keratan sulfate and chondroitin-6-sulfate are the GAGs, accumulating in MPS IVA. Since these are the main components of proteoglycans in bone and cartilage, MPS IVA is mainly a skeletal disease. ${ }^{19}$ Although, spinal cord compression can occur at all levels of the spine, beside kyphosis of the thoracolumbar region, compression of the spinal cord at the craniocervical junction, C1/C2 level accompanied by instability is most frequent. MPS IV patients have a high risk of having atlantoaxial instability. In the Natural History study, in 325 MPS IVA patients, odontoid dysplasia was described in $65 \%$, cervical spinal instability in $49 \%{ }^{25}$

For monitoring and diagnostics of spinal cord compression in MPS IVA, the following recommendations were published in $2013^{19}$ :

- Neurological examination at diagnosis and every 6 months.

- Plain radiography cervical spine (AP, lateral, neutral, and flexion-extension) at diagnosis and every 2 to 3 years.

- Plain radiography spine (AP and lateral) at diagnosis and every 2 to 3 years.

- MRI neutral position whole spine at diagnosis and every year.

- MRI of the cervical spine in flexion and extension at diagnosis and every 1 to 3 years.

- CT neutral position (region of interest) preoperative.

- The surgical approach is described below.

\section{Central Cord Syndrome in MPS VI}

The pathophysiology of craniocervical stenosis in MPS VI is very similar to MPS IVA, although instability is not as frequent as in MPS IVA. The following assessments are recommended ${ }^{26}$ :

- Neurological examination: At diagnosis, before enzyme replacement therapy (ERT) and 6 months after ERT starts, and interval of every 6 months.

- Plain radiography cervical spine (AP, lateral, neutral, and flexion-extension): > 1 year of age, diagnosis, before ERT, 1 year after ERT starts, and interval of every 3 years.

- CT in flexion and extension (if poor X-ray): > One year of age, diagnosis, before ERT, 1 year after ERT start, and interval of every 3 years.

- MRI in neutral position: >One year of age, diagnosis, before ERT, 1 year after ERT starts, and interval of every 1 to 2 years.

- MRI in flexion and extension: >One year of age, diagnosis, before ERT, 1 year after ERT starts, and interval of every 3 years.

- Evoked potentials: At diagnosis, before ERT start, 6 months after ERT starts, and interval of every 6 months to 1 year. 


\section{Central Cord Syndrome in MPS II}

There is not much literature about frequency of craniocervical stenosis in MPS II. However, an analysis of 719 patients enrolled in the Hunter Outcome Survey, described that the follow-up assessments concerning in MPS II and spinal cord compression is very poor. Only $12 \%$ of patients had $\geq 1$ cervical MR image, and only $22 \%$ had $\geq 1$ cervical X-ray image recorded. Only 21 patients (3\%) out of 683 had fusion or decompression surgery. In the Hunter Outcome Survey, it is reported that out of 39 patients with available imaging $68.8 \%$ showed cervical vertebral deformity, instability $3.1 \%$, and $9.4 \%$ spinal cord compression. Although, cervical spine stenosis in MPS II is not frequent as in other MPSs, it should be assessed regularly. ${ }^{10,27,28}$

\section{Central Cord Syndrome in MPS I}

It has been described that spinal cord compression is a complication in all types of MPS I patients and patients have to be screened regularly in the follow-up examinations. ${ }^{4}$

\section{Central Cord Syndrome in MPS III}

There is limited literature about MPS III and orthopedic complications. A study of 18 patients with MPS III with a median age of 10.3 years did not show any patient with craniocervical instability. ${ }^{29}$

\section{Central Cord Syndrome in MPS VII}

In a physicians' survey analyzing 56 MPS VII patients, 28 patients showed spinal cord compression. However, it was not defined on which level of the spinal cord. Almost 20\% of MPS VII patients received cervical fusion. ${ }^{12}$

\section{Kyphoscoliosis}

The kyphoscoliosis was considered previously a typical clinical sign to diagnose MPS patients. Gibbus deformity is present in almost all MPS types, almost all children with severe MPS I disease have it (-Fig. $\mathbf{3}$ ).

In a study of 19 MPS I patients treated with hematopoetic stem cell transplantation (HSCT), thoracolumbar kyphosis was clinically diagnosed in nearly $90 \%$ of patients at a mean age of 1.3 years, and was the first symptom of the disease in almost all patients; 58\% of the patients had kyphosis and scoliosis. The onset of kyphosis was 2.4 years, and the onset of scoliosis was 3.7 years. ${ }^{30}$

The causes of the malalignment are intervertebral disc degeneration and vertebral body dysplasia. Curves of $>40^{\circ}$ tend to progress and should be operated. A relative indication for surgery is a kyphosis of $>70^{\circ}$ and a scoliosis of $>50^{\circ}$, depending also on the aesthesia risk and complications/sufferance due to the deformity. Myelopathy is a strong indication for surgery. Mainly MPS I and VI show severe thoracolumbar kyphosis. The average age of surgery in MPS I patients is 8 years. $^{4,31}$

There is lack of information in the literature about kyphoscoliosis in MPS III beside one study enrolling 18 patients with MPS III (median age of 10.3 years), which showed that 3 patients had significant scoliosis and 2 others had L1 hypoplasia. ${ }^{29}$

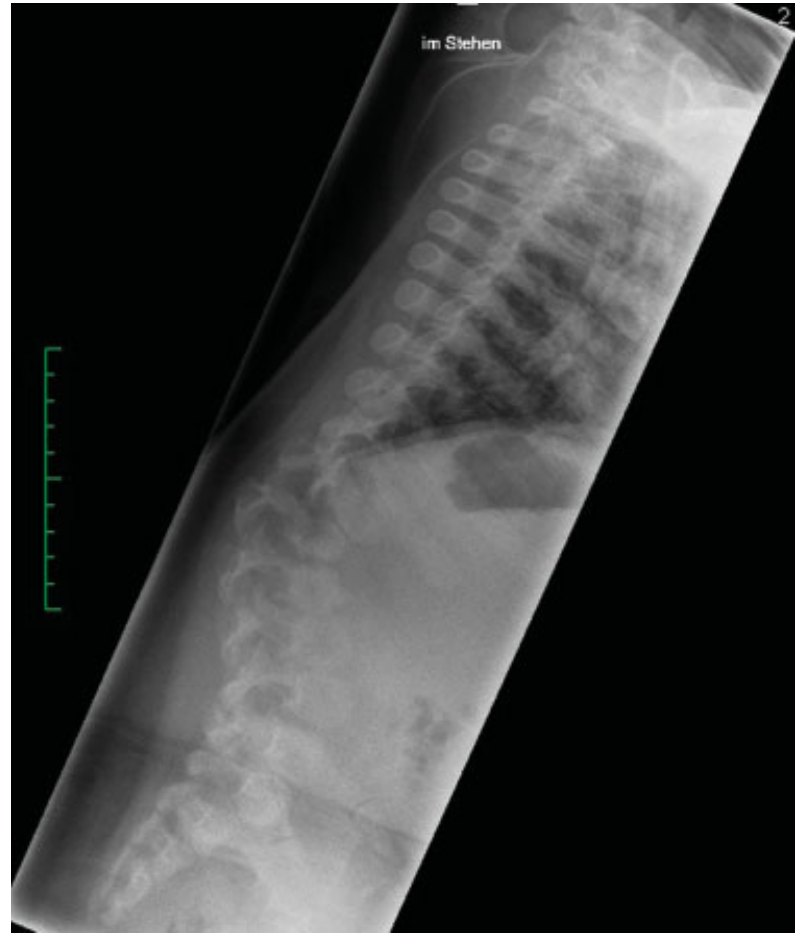

Fig. 3 X-ray of the lateral spine in a 7-year-old male MPS II patient with kyphoscoliosis. MPS, mucopolysaccharidosis.

In MPS II, only one case report is published. A 12-year-old boy with kyphoscoliosis was successfully treated with combined anterior/posterior instrumented arthrodesis. ${ }^{32}$ Based on available imaging, the evaluation of 39 patients enrolled in the Hunter Outcome Survey showed that in $87 \%$ any spine involvement was detected: almost $80 \%$ had thoracic vertebral deformity and 3.4\% spinal compression, while 94\% had lumbar vertebral deformity and 3\% spinal cord compression. Spine fusion or decompression was performed in less than $1 \%$ of patients.

In MPS VI kyphoscoliosis is mentioned as a disease complication but not described in detailed studies.

In the Natural History study of MPS IVA, 325 patients were enrolled, in which $13 \%$ patients exhibited thoracolumbar cord compression. ${ }^{25}$

In a physicians' survey of 56 MPS VII patients, almost 70\% showed kyphosis and scoliosis. Spine surgery was performed in around $15 \%$ of these patients. ${ }^{12}$

In our experience, stabilization of the deformity with a corset and surgical fixation after stop of growth may be an option. (Lampe, unpublished data)

The recommended surgical technique is anterior and posterior fusion and postoperative bracing for 3 to 6 months. Posterior stabilization and fusion only may lead to reoperation. Scoliosis operation follows the common treatment of scoliosis. $4,33,34$

There are no recommendations concerning the follow up of kyphoscoliosis but since the symptomatic is very similar to the cervical spinal cord compression, the same assessments should be used for diagnostics and in the follow-up: X-rays of the whole spine, AP and lateral in standing, MRI of 
the whole spine, and if available, somatosensory evoked potentials of the tibia nerve.

\section{Upper Limb Involvement}

\section{Carpal Tunnel Syndrome}

Carpal tunnel syndrome (CTS) is caused by compression of the median nerve at palmar side of the wrist. It presents with numbness and pain in the first three fingers and affects mainly women in the fifth and sixth decades of life. It is very uncommon in childhood and typical signs and symptoms are missing. CTS in childhood should always raise the suspicion of MPS. While it is uncommon in MPS III and IV, CTS has a high frequency in patients with MPS I, II, and VI. GAG accumulation in the retinaculum and the soft tissue around the median nerve in the carpal canal causes compression of the median nerve. It is important to diagnose and treat before irreversible damage of the nerve is present. The gold standard to detect CTS is to conduct regular nerve conduction studies, since early clinical signs and symptoms are mostly absent. In a study of 24 MPS patients (MPS I, II, and VI), ultrasound of the median nerve was analyzed as painless assessment to detect CTS. Results could confirm that nerve ultrasound has a high sensitivity and seemed to be more sensitive than clinical signs and nerve conduction velocity. ${ }^{35}$ However, no standard procedure for CTS diagnostics in MPS exists so far.

Different studies show the effect of HSCT in MPS I patients on CTS, but not of ERT. ${ }^{36}$

In MPS II, it has been recommended that standard electrophysiological testing should be initiated by 4 or 5 years of age and repeated at 1 or 2 years of intervals. ${ }^{37}$ Other results support this recommendation and advocate earlier initiation of electrophysiological studies by 3 years of age, as most of our patients older than 4 years already showed a severe degree of bilateral CTS. ${ }^{38}$

Studies have shown a prevalence of CTS up to $67 \%$ in patients with MPS I Scheie, ${ }^{39} 18 \%$ in patients with MPS II, ${ }^{40}$ and in 6 out of 7 patients with MPS VI. ${ }^{41} \mathrm{~A}$ new evaluation of 994 MPS I patients enrolled in the MPS I Registry report that 291 patients suffered from CTS. Median age at CTS diagnosis was 5.2 years for severe affected patients and 9.11 years in attenuated affected patients. ${ }^{42}$

The only available treatment is open surgical decompression of the transverse carpal ligament as well as debridement of hypertrophic tenosynovium. It has been shown, that nerve conduction velocity is not always improving although patients show clinical improvement. ${ }^{36}$

The greater the amount of axonal damage of the median nerve, the lower the recovery one can expect. Early recognition and intervention to ameliorate the symptoms are important in improving the QOL for MPS patients. ${ }^{36}$

\section{Trigger Finger}

Trigger fingers are seen frequently (MPS I, II, and VI) but must be carefully differentiated from joint contractures. In a study, 36 trigger fingers were operated with an A1 and/or A3 pulley release. One paper recommends partial resection of the flexor digitorum superficialis tendon and careful debridement of the tenosynovial deposits to reduce recurrence. ${ }^{8,43,44}$

\section{Growth}

Although height and weight in all MPSs are mainly in a normal range at birth, at a certain age, MPS patients leave their growth curve. ${ }^{45}$

In particular, severely affected MPSs show short stature. Dwarfism seems to be a combination of several factors as retardation of the endochondral ossification, structural deformities (genua valga, kyphoscoliosis) and endocrinopathy. Additionally, abnormalities of the thyroid, pituitary gland, and sex hormones may influence the growth in MPSs. ${ }^{46}$ Although, growth plate pathophysiology is poorly understood so far, it is evident, that GAG accumulation in the extracellular matrix as well as directly inside the lysosome leads to growth failure. Secondary effects of the storage are alteration of the signal transduction pathways as cell surface receptor activation, function and maintenance, modulation of humoral factor availability including cytokine and inflammatory modulator dysregulation, but also alteration the intracellular pathways, endocytosis, autophagy, and other lysosomal degradative pathways. Additionally, the GAG accumulation provokes an energy imbalance. ${ }^{45}$ The destroyed column organization of chondrocytes in the growth plate is shown in MPS I, IV and VI animal models and results in destroyed trabecular architecture.

Administration of growth hormone to improve growth, is still controversial. On the other hand, ERT seems to improve growth in MPS I, II, IV, and VI, as well as HSCT in MPS I.

Growth in HSC transplanted MPS I is strongly correlated with the age of transplantation. Usually, these patients have a normal growth up to 8 years of age before they leave their percentile. ${ }^{47}$ Enzyme replacement therapy, particularly started at an early age can improve growth velocity. MPS I ERT with Laronidase seems also to show improvement in height. In an observation of 5 patients over 6 years showed in prepubertal patients a gain of $33 \mathrm{~cm}$ in height. ${ }^{48}$ In contrast to that, a Polish study of 14 MPS I patients of ERT, compared with healthy population, did not show a significant gain of growth. ${ }^{49}$

In MPS II, growth decline is becoming present at the age of 8 to 10 years, which is so much later than in the other MPSs. Data of 133 patients aged 8 to 15 years on ERT with Elaprase were analyzed showing that there was a significant improvement on growth. The study also showed that patients with deletions/ large rearrangements/nonsense mutations as well as patients, which started ERT at an older age, had the highest growth deficit. ${ }^{50} \mathrm{~A}$ further study, which compared 13 patients that started ERT under the age of 6 years with $50 \mathrm{ERT}$ naïve patients, did not show any significant growth difference. ${ }^{51}$ The literature is inconsistent about growth and MPS II.

Growth data from 118 MPS III patients showed a deterioration of growth at the age of 6 years, mainly in the severe type, and leads to a final height of 2 SD below the normal age. ${ }^{11}$

In MPS IVA, growth is used as a sign of disease severity. In the Natural History study of 325 MPS IVA patients, the mean age was 14.5 years. Mean \pm standard deviation of $z$-scores 
were:5.6 \pm 3.1. Data from the international Morquio registry, enrolled 326 patients and the final adult height for affected males and females was $122.5 \pm 22.5 \mathrm{~cm}$ and $116.5 \pm 20.5 \mathrm{~cm}$, respectively. ${ }^{52}$ In the phase II trial of 15 MPS IVA patients less than 5 years of age treated with Elosulfase over 52 weeks, the mean $z$-score of these cohort improved from -0.6 to -0.4 . $^{53}$

In MPS VI, data of 229 patients (under the age of 25 years) pre-ERT were analyzed to show the natural history of growth in MPS VI. At the age of 2 to 3 years, patients decline in growth. The fast progressing patients reach a plateau at the age of 10 years. The median height of fast progressing MPS VI patients at the age of 18 years was $109.3 \mathrm{~cm}$, in contrast to the slowly progressing MPS VI patients with a final height of $144.1 \mathrm{~cm} .{ }^{54}$

An investigation, confirming the higher growth rate in MPS VI on ERT was published in 2017. This study showed that depending on the baseline urinary GAG levels and age at ERT initiation, younger MPS VI patients treated with ERT showed a significant increase of height, measured by $z$-scores. Most benefit in growth was shown in patients who started ERT $<6$ years of age and had high urinary GAGs at baseline. On the other hand, patients who started ERT $>15$ years of age did not show any benefit on growth, independently from the baseline GAGs. ${ }^{55}$

\section{Conflict of Interest}

None.

\section{References}

1 Bonafe L, Cormier-Daire V, Hall C, et al. Nosology and classification of genetic skeletal disorders: 2015 revision. Am J Med Genet A 2015;167A(12):2869-2892

2 Clarke LA, Hollak CE. The clinical spectrum and pathophysiology of skeletal complications in lysosomal storage disorders. Best Pract Res Clin Endocrinol Metab 2015;29(02):219-235

3 White KK, Harmatz P. Orthopedic management of mucopolysaccharide disease. J Pediatr Rehabil Med 2010;3(01):47-56

4 White KK. Orthopaedic aspects of mucopolysaccharidoses. Rheumatology (Oxford) 2011;50(Suppl 5):v26-v33

5 White KK, Jester A, Bache CE, et al. Orthopedic management of the extremities in patients with Morquio A syndrome. J Child Orthop 2014;8(04):295-304

6 Langereis EJ, Borgo A, Crushell E, et al. Treatment of hip dysplasia in patients with mucopolysaccharidosis type I after hematopoietic stem cell transplantation: results of an international consensus procedure. Orphanet J Rare Dis 2013;8:155

7 Harris WH. Traumatic arthritis of the hip after dislocation and acetabular fractures: treatment by mold arthroplasty. An endresult study using a new method of result evaluation. J Bone Joint Surg Am 1969;51(04):737-755

8 Williams N, Challoumas D, Eastwood DM. Does orthopaedic surgery improve quality of life and function in patients with mucopolysaccharidoses? . J Child Orthop 2017;11(04): 289-297

9 Ashby E, Baker M, Eastwood DM. Characterization of hip morphology in children with mucopolysaccharidosistypes I and II. J Pediatr Orthop 2016;36(04):370-375

10 Link B, de Camargo Pinto LL, Giugliani R, et al. Orthopedic manifestations in patients with mucopolysaccharidosis type II (Hunter syndrome) enrolled in the Hunter Outcome Survey. Orthop Rev (Pavia) 2010;2(02):e16

11 de Ruijter J, Broere L, Mulder MF, et al. Growth in patients with mucopolysaccharidosis type III (Sanfilippo disease). J Inherit Metab Dis 2014;37(03):447-454
12 Montaño AM, Lock-Hock N, Steiner RD, et al. Clinical course of sly syndrome (mucopolysaccharidosis type VII). J Med Genet 2016; 53(06):403-418

13 Oussoren E, Bessems JHJM, Pollet V, et al. A long term follow-up study of the development of hip disease in Mucopolysaccharidosis type VI. Mol Genet Metab 2017;121(03):241-251

14 Dhawale AA, Johari AN, Nemade A. Hip dislocation during lengthening of congenital short femur. J PediatrOrthop B 2012; 21(03):240-247

15 Lewis JR, Gibson PH. Bilateral hip replacement in three patients with lysosomal storage disease: Mucopolysaccharidosis type IV and Mucolipidosis type III. J Bone Joint Surg Br 2010;92(02):289-292

16 Montaño AM, Tomatsu S, Gottesman GS, Smith M, Orii T. International MorquioA Registry: clinical manifestation and natural course of Morquio A disease. J Inherit Metab Dis 2007;30(02):165-174

17 Garcia P, Sousa SB, Ling TP, et al. Skeletal complications in mucopolysaccharidosis VI patients: Case reports. J PediatrRehabil Med 2010;3(01):63-69

18 Kennedy J, Noel J, O'Meara A, Kelly P. Foot and ankle abnormalities in the Hurler syndrome: additions to the phenotype. J Pediatr Orthop 2013;33(05):558-562

19 Solanki GA, Martin KW, Theroux MC, et al. Spinal involvement in mucopolysaccharidosis IVA (Morquio-Brailsford or Morquio A syndrome): presentation, diagnosis and management. J Inherit Metab Dis 2013;36(02):339-355

20 Solanki GA, Alden TD, Burton BK, et al. A multinational, multidisciplinary consensus for the diagnosis and management of spinal cord compression among patients with mucopolysaccharidosis VI. Mol Genet Metab 2012;107(1-2):15-24

21 Horovitz DD, Magalhães TS, Pena e Costa A, et al. Spinal cord compression in young children with type VI mucopolysaccharidosis. Mol Genet Metab 2011;104(03):295-300

22 Lampe C, Lampe C, Schwarz M, Müller-Forell W, Harmatz P, Mengel E. Craniocervical decompression in patients with mucopolysaccharidosis VI: development of a scoring system to determine indication and outcome of surgery. J Inherit Metab Dis 2013; 36(06):1005-1013

23 Möllmann C, Lampe CG, Müller-Forell W, et al. Development of a scoring system to evaluate the severity of craniocervical spinal cord compression in patients with mucopolysaccharidosis IVA (Morquio A Syndrome). JIMD Repv 2013;11:65-72

24 Reichert R, Campos LG, Vairo F, et al. Neuroimaging findings in patients with mucopolysaccharidosis: what you really need to know. Radiographics 2016;36(05):1448-1462

25 Harmatz P, Mengel KE, Giugliani R, et al. The Morquio A clinical assessment program: baseline results illustrating progressive, multisystemic clinical impairments in Morquio A subjects. Mol Genet Metab 2013;109(01):54-61

26 Solanki GA, Sun PP, Martin KW, et al; CSP Study Group. Cervical cord compression in mucopolysaccharidosis VI (MPS VI): findings from the MPS VI Clinical Surveillance Program (CSP). Mol Genet Metab 2016;118(04):310-318

27 Harmatz P, Lampe C, Burton BK, Mendelsohn NJ, Fang J. Cervical spine pathology in Hunter syndrome: data from the Hunter Outcome Survey. Mol Genet Metab 2013;108(02):S46-S47

28 Manara R, Priante E, Grimaldi M, et al. Brain and spine MRI features of Hunter disease: frequency, natural evolution and response to therapy. J Inherit Metab Dis 2011;34(03):763-780

29 White KK, Karol LA, White DR, Hale S. Musculoskeletal manifestations of Sanfilippo Syndrome (mucopolysaccharidosis type III). J Pediatr Orthop 2011;31(05):594-598

30 Schmidt M, Breyer S, Löbel U, et al. Musculoskeletal manifestations in mucopolysaccharidosis type I (Hurler syndrome) following hematopoietic stem cell transplantation. Orphanet J Rare Dis 2016;11(01):93

31 Abelin Genevois K, Garin C, Solla F, Guffon N, Kohler R. Surgical management of thoracolumbar kyphosis in mucopolysaccharidosis type 1 in a reference center. J Inherit Metab Dis 2014;37(01):69-78 
32 Roberts SB, Tsirikos AI. Thoracolumbar kyphoscoliosis with unilateral subluxation of the spine and postoperative lumbar spondylolisthesis in Hunter syndrome. J Neurosurg Spine 2016;24 (03):402-406

33 Garrido E, Tomé-Bermejo F, Adams CI. Combined spinal arthrodesis with instrumentation for the management of progressive thoracolumbar kyphosis in children with mucopolysaccharidosis. Eur Spine J 2014;23(12):2751-2757

34 Roberts SB, Dryden R, Tsirikos AI. Thoracolumbar kyphosis in patients with mucopolysaccharidoses: clinical outcomes and predictive radiographic factors for progression of deformity. Bone Joint J 2016;98-B(02):229-237

35 Bäumer T, Bühring N, Schelle T, Münchau A, Muschol N. Nerve ultrasound in clinical management of carpal tunnel syndrome in mucopolysaccharidosis. Dev Med Child Neurol 2016;58(11): 1172-1179

36 White KK. Carpal tunnel syndrome in mucopolysaccharidosis: the value of rare disease registries. Dev Med Child Neurol 2017;59 (12):1213-1214

37 Muenzer J, Beck M, Eng CM, et al. Multidisciplinary management of Hunter syndrome. Pediatrics 2009;124(06):e1228-e1239

38 Kwon JY, Ko K, Sohn YB, et al. High prevalence of carpal tunnel syndrome in children with mucopolysaccharidosis type II (Hunter syndrome). Am J Med Genet A 2011;155A(06):1329-1335

39 Thomas JA, Beck M, Clarke JT, Cox GF. Childhood onset of Scheie syndrome, the attenuated form of mucopolysaccharidosis I.J Inherit Metab Dis 2010;33(04):421-427

40 Mendelsohn NJ, Harmatz P, Bodamer O, et al; Hunter Outcome Survey Investigators. Importance of surgical history in diagnosing mucopolysaccharidosis type II (Hunter syndrome): data from the Hunter Outcome Survey. Genet Med 2010;12(12): 816-822

41 Haddad FS, Jones DH, Vellodi A, Kane N, Pitt MC. Carpal tunnel syndrome in the mucopolysaccharidoses and mucolipidoses. J Bone Joint Surg Br 1997;79(04):576-582

42 Viskochil D, Muenzer J, Guffon N, et al. Carpal tunnel syndrome in mucopolysaccharidosis I: a registry-based cohort study. Dev Med Child Neurol 2017;59(12):1269-1275

43 Morishita K, Petty RE. Musculoskeletal manifestations of mucopolysaccharidoses. Rheumatology (Oxford) 2011;50(Suppl 5): v19-v25

44 Van Heest AE, House J, Krivit W, Walker K. Surgical treatment of carpal tunnel syndrome and trigger digits in children with mucopolysaccharide storage disorders. J Hand Surg Am 1998;23 (02):236-243

45 Clarke LA. Pathogenesis of skeletal and connective tissue involvement in the mucopolysaccharidoses: glycosaminoglycan storage is merely the instigator. Rheumatology (Oxford) 2011;50 (Suppl 5):v13-v18

46 Polgreen LE, Tolar J, Plog M, et al. Growth and endocrine function in patients with Hurler syndrome after hematopoietic stem cell transplantation. Bone Marrow Transplant 2008;41(12):1005-1011

47 Vellodi A, Young EP, Cooper A, et al. Bone marrow transplantation for mucopolysaccharidosis type I: experience of two British centres. Arch Dis Child 1997;76(02):92-99

48 Sifuentes M, Doroshow R, Hoft R, et al. A follow-up study of MPS I patients treated with laronidase enzyme replacement therapy for 6 years. Mol Genet Metab 2007;90(02):171-180

49 Tylki-Szymanska A, Rozdzynska A, Jurecka A, Marucha J, Czartoryska B. Anthropometric data of 14 patients with mucopolysaccharidosis I: retrospective analysis and efficacy of recombinant human alpha-L-iduronidase (laronidase). Mol Genet Metab 2010; 99(01):10-17

50 Jones SA, Parini R, Harmatz P, Giugliani R, Fang J, Mendelsohn NJ; HOS Natural History Working Group on behalf of HOS Investigators. The effect of idursulfase on growth in patients with Hunter syndrome: data from the Hunter Outcome Survey (HOS). Mol Genet Metab 2013;109(01):41-48

51 Żuber Z, Różdżyńska-Świątkowska A, Jurecka A, Tylki-Szymańska A. The effect of recombinant human iduronate-2-sulfatase (Idursulfase) on growth in young patients with mucopolysaccharidosis type II. PLoS One 2014;9(01):e85074

52 Montaño AM, Tomatsu S, Brusius A, Smith M, Orii T. Growth charts for patients affected with Morquio A disease. Am J Med Genet A 2008;146A(10):1286-1295

53 Jones SA, Bialer M, Parini R, et al. Safety and clinical activity of elosulfasealfa in pediatric patients with Morquio A syndrome (mucopolysaccharidosis IVA) less than 5 y. Pediatr Res 2015;78 (06):717-722

54 Quartel A, Hendriksz CJ, Parini R, Graham S, Lin P, Harmatz P. Growth Charts for Individuals with Mucopolysaccharidosis VI (Maroteaux-Lamy Syndrome). JIMD Rep 2015;18:1-11

55 Harmatz P, Hendriksz CJ, Lampe C, et al; MPS VI Study Group. The effect of galsulfase enzyme replacement therapy on the growth of patients with mucopolysaccharidosis VI (Maroteaux-Lamy syndrome). Mol Genet Metab 2017;122(1-2):107-112 